\title{
Lessons from archaeology and heritage studies for the long-term preservation of records, knowledge and memory concerning deep geological disposal sites for nuclear waste
}

\author{
Cornelius Holtorf and Anders Högberg \\ UNESCO Chair on Heritage Futures, Linnaeus University, Kalmar, Sweden \\ Correspondence: Cornelius Holtorf (cornelius.holtorf@lnu.se) and \\ Anders Högberg (anders.hogberg@lnu.se)
}

Published: 10 November 2021

\begin{abstract}
Safe disposal of nuclear waste in deep geological repositories requires secure knowledge transfer or knowledge recovery in time spans of many tens of thousands of years. Never before has any detailed record, knowledge or memory been reliably preserved or recovered over comparable time periods.

This challenge has been extensively addressed since the late 1980s, initially during the SANDIA workshops in the USA and more recently in the Nuclear Energy Agency/Organisation for Economic Co-operation and Development (NEA/OECD) project on Preservation of Records, Knowledge and Memory Across Generations (Schröder, 2019). Experts from many disciplines including engineering, the natural sciences, information technology, social studies of science and technology, semiotics, public management, and design as well as artists have contributed to these discussions. Some scholars from the humanities have been involved in working on these issues, especially in recent decades. At the same time, much of the existing work has drawn on assumptions about human history, archaeological monuments and cultural heritage that have been scrutinized and deemed deeply flawed by Joyce (2020).

The authors of the present paper are archaeologists and cultural heritage experts. For the past decade, they have been working with the challenge of preserving records, knowledge and memory concerning deep geological disposal sites for nuclear waste (Holtorf and Högberg, 2021). From the perspective of the human sciences, in particular archaeology and heritage studies, the unique task at hand involves not only the previously recognized challenges that require consideration of long-term material durability, linguistic intelligibility, and appropriate sense-making of any communicated information but also two challenges not previously addressed:
\end{abstract}

1. Human action as informed by cultural and social processes. In designing of various long-term mechanisms, we risk overlooking that what people will do is not going to be governed by mechanics. How human beings learn, reason, value, decide, and act is informed by specific cultural and social processes creating context and meaning. We must avoid ignoring these complexities governing human thinking and agency. This challenge requires more work on understanding how sentient and intelligent beings like humans act in variable contexts across time and space.

2. Our anticipatory assumptions. A proverb states that "nothing ages faster than the future". In making assumptions about future generations' understandings, meanings, and significances of our nuclear waste we risk "colonizing" the future, fail to embrace variability over time, and miss realizing multiple futures and emerging conditions. We must therefore not foreclose uncertain futures but instead create circumstances favorable for change and transformation of relevant knowledge and memory. This challenge requires more work with processes of translation between generations. 
The challenges of assessing our anticipatory assumptions and understanding how humans act will also need to be addressed in transmitting records, knowledge and memory for the benefit of future generations.

Kurzfassung. Die sichere Entsorgung nuklearer Abfälle in geologischen Tiefenlagern erfordert einen sicheren Wissenstransfer bzw. eine Wissenswiederherstellung über Zeitspannen von vielen zehntausend Jahren. Nie zuvor konnten detaillierte Aufzeichnungen, Wissen oder Erinnerungen über vergleichbare Zeiträume zuverlässig erhalten oder wiederhergestellt werden.

Mit dieser Herausforderung hat man sich seit Ende der 1980er Jahre eingehend befasst, zunächst im Rahmen der SANDIA-Workshops in den USA und in jüngerer Zeit im Rahmen des NEA/OECD-Projekts (Nuclear Energy Agency/Organisation for Economic Co-operation and Development) zur Bewahrung von Aufzeichnungen, Wissen und Erinnerungen über Generationen hinweg (Schröder, 2019). Experten aus vielen Fachbereichen, darunter Ingenieur- und Naturwissenschaften, Informatik, Wissenschafts- und Techniksoziologie, Semiotik, Public Management und Design, sowie Künstler haben sich an diesen Diskussionen beteiligt. Einige Humanwissenschaftler*innen haben sich ebenfalls mit diesen Fragen befasst, insbesondere in den letzten Jahrzehnten. Gleichzeitig stützt sich ein Großteil der existierenden Arbeiten auf Hypothesen zur menschlichen Geschichte, zu archäologischen Denkmälern und zum kulturellen Erbe, welche von Joyce (2020) hinterfragt und als äußerst problematisch beurteilt wurden.

Die Autoren der vorliegenden Arbeit sind Archäologen und Experten für kulturelles Erbe. In den letzten zehn Jahren haben sie sich mit der Herausforderung befasst, Aufzeichnungen, Wissen und Erinnerungen im Zusammenhang mit geologischen Tiefenlagern für Atommüll zu bewahren (Holtorf und Högberg, 2021). Aus Sicht der Humanwissenschaften, insbesondere der Archäologie und der Kulturerbeforschung, beinhaltet diese einzigartige Aufgabe nicht nur die bereits bekannten Herausforderungen, die eine Berücksichtigung der langfristigen materiellen Haltbarkeit, der sprachlichen Verständlichkeit und der angemessenen Sinngebung jeglicher kommunizierten Informationen erfordern, sondern auch zwei bisher nicht berücksichtigte Herausforderungen:

1. Menschliches Handeln, das durch kulturelle und soziale Prozesse geprägt ist. Bei der Entwicklung verschiedener langfristiger Mechanismen besteht die Gefahr, dass wir übersehen, dass das, was Menschen tun werden, nicht von Mechanik bestimmt wird. Die Art und Weise, wie Menschen lernen, argumentieren, bewerten, entscheiden und handeln, wird durch spezifische kulturelle und soziale Prozesse bestimmt, die Kontext und Bedeutung schaffen. Wir dürfen diese komplexen Zusammenhänge, die das menschliche Denken und Handeln bestimmen, nicht ignorieren. Diese Herausforderung erfordert noch mehr Arbeit, um zu verstehen, wie empfindungsfähige und intelligente Wesen wie der Mensch in unterschiedlichen Kontexten über Zeit und Raum hinweg handeln.

2. Unsere antizipatorischen Annahmen. Ein Sprichwort besagt, dass „nichts schneller altert als die Zukunft“. Wenn wir Hypothesen über das Verständnis, die Bedeutung und den Stellenwert unseres „Atommülls“ für künftige Generationen aufstellen, riskieren wir, die Zukunft zu „kolonisieren“, versäumen es, Variabilität im Laufe der Zeit zu berücksichtigen, und verpassen es, vielfältige Zukünfte und neuentstehende Bedingungen zu erkennen. Wir dürfen daher ungewisse Zukünfte nicht ausschließen, sondern müssen stattdessen günstige Bedingungen für Wandel und Transformation von relevantem Wissen und Erinnerung schaffen. Diese Herausforderung erfordert eine stärkere Beschäftigung mit Übersetzungsprozessen zwischen den Generationen.

Die Herausforderungen der Bewertung unserer antizipatorischen Annahmen und des Verständnisses des menschlichen Handelns müssen auch bei der Weitergabe von Aufzeichnungen, Wissen und Erinnerungen zum Nutzen künftiger Generationen angegangen werden.

\section{References}

Holtorf, C. and Högberg, A.: What lies ahead? Nuclear waste as cultural heritage of the future, in: Cultural Heritage and the Future, edited by: Holtorf, C and Högberg, A., Routledge, London, New York, 144-158, 2021.
Joyce, R.: The Future of Nuclear Waste: What Art and Archaeology Can Tell Us About Securing the World's Most Hazardous Waste, Oxford University Press, Oxford, 2020.

Schröder, J.: Preservation of Records, Knowledge and Memory (RK\&M) Across Generations: Final Report of the RK\&M Initiative, OECD, Nuclear Energy Agency, Paris, available at: https: //www.oecd-nea.org/rwm/pubs/2019/7421-RKM-Final.pdf (last access: 9 June 2021), 2019. 